\title{
A Study on the Use Strategies of Chinese Characters in the Chinese-Korean Translation
}

\author{
Liang Zhao \\ Foreign Language College, Beihua University, Jilin City, Jilin Province, \\ 132013, China
}

\begin{abstract}
Chinese characters have been used by people on the Korean Peninsula for a long time. The Chinese characters and vocabulary are used extensively in ancient and modern Korean. Exchanges between people in China and Korea enjoy a long history. Besides, language and cultural contacts are frequent, and Korean and Chinese characters also have profound origins. Based on the author's learning and practical experience, this paper compared Chinese characters in Korean with the meaning expression and emotional coloring of modern Chinese language. Then, the article discussed characteristics of Chinese characters in Korean and the translation methods.

Keywords: Chinese characters; Chinese-Korean translation; Use strategies
\end{abstract}

\section{Introduction}

The Korean Peninsula has used Chinese characters for a long time, which results in that a large number of Chinese characters flow into Korean. In consideration of clear expression, the article calls Chinese words in Korean as Chinese characters, while the Chinese vocabulary is referred to as Chinese words in the following content. Chinese words integrate with vernacular words in Korean, which constitutes the basic vocabulary system of Korean. Although South Korea and North Korea which are located in the Korean Peninsula have carried out language reform to maintain the purity and independence of national languages, they just removed the Chinese characters in terms of writing, and the vocabulary meaning and the use of Chinese vocabulary remain the same[1]. 


\section{Chinese Characters in Korean}

\subsection{Types of words}

There are rich vocabulary and various expressions in Chinese and Korean. In terms of classification of words, Korean words include Chinese characters, vernacular words and foreign words. A large number of Chinese words are introduced into the Korean Peninsula and integrated with vernacular words in Korean which constitutes the basic vocabulary system of Korean. Chinese characters refer to words which are created based on Chinese words. They account for sixty to seventy percent of the Korean vocabulary. Therefore, the translation of Chinese characters is a key to word translation. There are three kinds of Chinese characters in Korean. The first kind is created by the ancient Korean by virtue of the pronunciation, form and meaning of Chinese characters, such as: - friend and - fresh fish. The other kind is words borrowed directly from China or words that are revised little, such as: - parents, - oneself and - brave[2].

\subsection{The structural features of Chinese characters and error-prone points}

The Chinese characters in Korean has the same structure with Chinese words, such as - time and - poem. Korean language reform replaced the use of Chinese characters with Korean, which causes difficulty in understanding Chinese characters. Besides, there are some mistakes in the translation. For example, may be translated into forest arson. However, it may actually be the opposite, which is the forest fire prevention. may be translated into Korean cattle, and it may refer to the shipyard. The lexical meaning, application method and emotional coloring of Chinese characters in Korean are different from modern Chinese language. In view of this, it is easy for translators to make translation mistakes, if they can not understand the Chinese characters based on the context.

\subsection{The significance of the mastery of Chinese characters for translation}

At present, there are 1800 Chinese characters can be used in Korean legally. The first problem in the translation between Chinese and Korean is the accurate grasp and application of the meaning of Chinese Characters. In the translation, it has a great significance to grasp the regularity, the meaning changes and evolution characteristics of Chinese characters for ensuring the accuracy, quality and speed of the translation. 


\section{The Cause of Differences between Chinese Characters and Their Cognate Chinese Words}

\subsection{The reason analysis of usage difference}

In the reason analysis of usage difference, Chinese is an independent language and Korean is an adhesive language according to morphological changes. The independent language lacks inflexion; that a word belongs to which element in the sentence is mainly determined by the word order. Adhesive language has a lot of bound morphemes with extra meaning; that a word belongs to which element in the sentence is mainly determined by the additional morphemes. When Chinese words are borrowed into the Korean language system, they need to adopt to the Korean situation[3]. Therefore, it cause a difference in the usage and morphology between the original Chinese words and Chinese characters in Korean. The original grammatical meaning is weakened and these words need to add other morphemes to express the corresponding meaning. When they appear in the sentence simultaneously, translators need to understand these words in accordance with the context and different morphemes.

\subsection{The reason analysis of semantic difference}

Each language has its own complete and independent vocabulary system. As a complex organism, the vocabulary system is not changeless and its interior has been changing and maintaining a dynamic balance. In the vocabulary system, all vocabulary is in a specific position and assumes a certain task to express meaning, which has an organic link with other vocabulary. This connection is not fixed and it keeps changing and developing with the development of the society, which is influenced by the history, culture, national psychology and customs. When Chinese characters are borrowed into Korean, they and their cognate Chinese words are in two different vocabulary systems which keeps constantly changing and developing. Therefore, Chinese characters and their cognate Chinese words have many semantic differences[4].

\section{The Translation Methods of Chinese Characters in Modern Korean}

\subsection{Literal translation}

Literal translation is one of the basic translation methods, which is a translation method that uses fluent and legible words to express the original information as far as possible on the premise of embodying the original content, form and style. The literal translation of Chinese characters is the direct use of Chinese words that Chinese characters literally represent to complete the translation. Literal translation is very important in the translation of Chinese characters. It is used for those Chinese characters that have the same constituent elements, structure and 
meanings as the cognate Chinese words, or for the Chinese characters with the same elements and opposite structure as the Chinese words. However, in the literal translation, Chinese characters must have the exactly same literal meanings with its cognate Chinese words so as to ensure the correct translation.

\subsection{Free translation}

Although there are many Chinese characters in Korean, Korean and Chinese belong to different language families. Besides, due to differences in nations and living environment, there exist many differences between the two languages. These differences determine the use of free translation between Chinese and Korean translation. Translation requires the translator to faithfully and accurately express the original meaning, so that the targeted readers will have the same feeling with readers who read the original text. However, differences bring many obstacles to the translation work. When this obstacle can not be solved by literal translation, we need to use the method of accommodation and compensation to translate the original text, which is the main basis for the free translation. Free translation is one of the basic translation methods. In order to accurately reproduce the meaning of the original text, the translation accommodates and compensates the original text[5]. However, this kind of accommodation and compensation is not to modify, increase or decrease words arbitrarily. In the translation of Chinese characters, free translation and literal translation are equally important. It can make the translation more in line with the norms of Chinese and can express the information of Chinese characters in the original text faithfully and accurately.

\subsection{Extended translation and invert translation}

The extended meaning is a new meaning of the original text. The so-called extended translation means to achieve the new meaning of the word. It is to accurately translate words in the original text by virtue of the translator's processing. The purpose of this translation method is to make the translation logical and straighten the matching relationship to achieve a better expression of the original text. However, translators should not translate the text arbitrarily. The extended translation of Chinese characters is a method to define the meaning of Chinese characters with new meanings in the context .

Compared to the extension, the semantic transformation is a greater extension. The meaning of a word in a specific language environment will produce augmented meaning, expansion, transformation and variation, so translators not only need to extend the meaning, but also need to use the invert translation sometimes. The so-called invert translation is to transform and change the original expression by virtue of of augmented and extended meaning with transformation and variation in order to straighten out the collocations with the central word. Therefore, if Chinese characters in the original text have these features, translators can use invert translation method. The purpose of this method is to make the translation reasonable, logical and corresponding to the expression habits. 


\subsection{Addition and omission translation}

In Chinese-Korean or Korean-Chinese translation, in order to pursue the effect of the translation, after the scrutiny of the language in the original text, the translation will increase or decrease words inevitably. From the perspective of information science, the original information includes text information and scene information. In the translation, because of the existence of scene information in the original text, such as the sender, receiver, communication theme, communication time, space, emotion, action and atmosphere, translators need to add the scene information to the translation according to the targeted language form, so there is an increase in the content of the translation[6]. When the translator has grasped the scene information in the original text, it is unnecessary to express all original information with words, so some information in the translation content is reduced. Chinese and Korean have their own characteristics, so in the translation between the two languages, it is necessary to increase or decrease some words in the re-processing of the original information. Although the majority of Chinese characters in Korean have negate relationship with Chinese words, they have changed much, because Chinese characters have been used in Korean for a long time. Therefore, word information needs to replace and compensate with scene information in order to make the original information equal to the translation information, which leads to the increase and decrease of vocabulary.

\subsection{Restitution translation}

Restitution translation is used in the translation of idioms and proverbs. The socalled restitution translation of Chinese characters refers to that translators translate Chinese characters with the same structure and meaning as the Chinese negate words into Chinese idioms[7]. This method in the translation of Chinese characters is mainly used to replace Korean idioms and dialects with specific elements and Chinese characters with the same structure and opposite meanings

\subsection{Converting Translation}

Converting translation refers to that translators use the conversion of word class, coloring, image and angle to make the translation more appropriate, vivid and logical. In the translation of Chinese characters in Korean, translators need to transform words, which is mainly the conversion of the class and coloring of the Chinese characters. Conversion of word classes means to convert Chinese characters between nouns and verbs or nouns and adjectives in translation. Conversion of coloring is conversion of the coloring of Chinese characters in the translation, which is based on the different language environment. In fact, it is a reflection of the scene information, which is that the scene information in the original content is converted into text messages. 


\section{Conclusion}

In view of the large proportion of Chinese characters in Korean, the correct translation of Chinese characters and the mastery of translation skills is a necessary link to make the correct translation of sentences, paragraphs and articles. At the same time, learning vocabulary well is a key to overcome the main obstacles in translation. This requires translators to study and accumulate words in the daily translation constantly, master the characteristics of words in the translation and seek the equivalent expression pattern of the two languages.

\section{Reference}

[1] Zheng Xing. A Discussion on the Translation of Chinese Characters in Korean. Vocational Education (On Trimonthly Publication), (11), pp.18-21, 2015. [2] Liu Suzhen. An Analysis of the Characteristics and Translation Laws of Chinese Characters in Korean. Journal of Jilin Radio and Television University, (05), pp.143-144, 2015.

[3] Cui Yingshuang. A Discussion on the Translation of Chinese Characters. Science and Technology Information, (07), pp.198-199, 2010.

[4] Pu Jinfeng. An Exploration of Chinese Characters in Korean and Its Teaching Methods. Journal of Anhui University of Technology (Social Science Edition), (01), pp.125-126, 2010.

[5] Jin Shan. A Discussion on the Teaching of Chinese Characters in Korean. Science and Technology Information, (34), pp.524-525, 2009.

[6] Zhao Yunjing. A Research of Chinese Characters in Korean and Its Teaching. Fudan Forum on Foreign Language and Literature, (01), pp.136-140, 2009.

[7] Chen Yanmin. A Study of the Migration of Isomorphic Chinese Characters in the Chinese-Korean Translation. Journal of Educational Institute in Jilin Province, (01), pp.153-154, 2009. 